Pacific Journal of Mathematics

Low Dnersooxal ManuaL SE B: 


\title{
ON LOW DIMENSIONAL MINIMAL SETS
}

\author{
SoON-KYU KIM
}

Let $(X, G, f)$ be a topological transformation group. Suppose that the phase space $X$ is compact, separable metric, and locally contractible and the group $G$ is the additive group of all real numbers $R$ with the usual topology. If $X$ is a minimal set of $\operatorname{dim}_{L}(X) \leqq 2$ then $X$ is a manifold, imposing a further condition on the action when $\operatorname{dim}_{L}(X)=2$. Hence $X$ is a singleton, a circle or a torus according to its dimension.

A topological transformation group is a triple $(X, G, f)$ consisting of a topological space $X$, a topological group $G$, and a continuous map $f$ from $G \times X$ into $X$ such that $f(e, x)=x, f(h, f(g, x))=f(g h, x)$ for any $x$ in $X$ and any $g, h$ in $G$ and the identity element $e$ of $G$.

The phase space $X$ of a topological transformation group $(X, G, f)$ is called a minimal set if for each $x \in X$ the closure of the orbit of $x$ is $X$ itself. A locally contractible space $X$ is a space such that for each $x \in X$ and for any open set $U$ containing $x$ there is an open set $V$ containing $x$, which is contractible in $U$ to the point $x$.

Chu [3] has shown that if the phase space $X$ is a compact Hausdorff minimal set and $\operatorname{dim}_{L}(X) \leqq n$, then $H^{n}(A, L)=0$ for every proper closed subset $A$ of $X$ under any connected topological group $G$. Here $\operatorname{dim}_{L}(X)$ is the cohomology dimension of $X$ in the sense of Cohen ([2], [4]) and $L$ is a principal ideal domain. The Alexander-Spanier cohomology theory is used here. Using this result, Chu has answered questions that were raised by Gottschalk [6]. He proved that the universal curve of Menger and the universal curve of Sierpinski are not minimal sets under any connected topological group.

Chu has also shown that some cohomological natures of a minimal set are similar to those of a generalized manifold. We try to see whether certain minimal sets are actually generalized manifolds. In this regard, we have some results in low dimensions as mentioned in the abstract.

We use the section theorem of Bebutov and Hájek and the umbrella theorem of Bing-Borsuk that we state here.

The section theorem ([11: p. 332] and [8: p. 210])

Given a topological transformation group $(X, R, f)$ with $X$ separable metric and a non-fixed point $x_{0}$ in $X$ there exist sections $S \ni x_{0}$ generating arbitrary small neighborhoods of $x_{0}$ in $X$. If $X$ is locally compact or locally connected, then $S$ may be taken compact or connected respectively. Furthermore, if $X$ is compact and locally connected, then $S$ may be taken locally connected. 
The umbrella theorem ([1: Cor. 5.3]).

In an $n$-dimensional locally contractible separable metric space $X$ the set of all centers of $n$-dimensional umbrellas (see [1] for definition) contained in $X$ is of the first category of Baire.

We note that if the phase group is discrete then $X$ is not necessarily a homogeneous space hence not a manifold ([5], [6: p. 139]).

\section{Zero and one dimensional minimal sets.}

THEOREM 1. Let $(X, R, f)$ be a topological transformation group with $X$ a locally connected compact separable metric space and $R$ the additive real group. Suppose $X$ is a minimal set of $\operatorname{dim}_{L}(X)=0$ or 1. Then $X$ is a singleton or a circle.

Proof. Since $X$ is necessarily connected, $X$ is a point if $\operatorname{dim}_{L}(X)=0$. Let the dimension of $X$ be 1 . Since each point $x \in X$ is not a fixed point, by the section theorem of Bebutov and Hájek there is a section generating arbitrary small neighborhoods of $x$ in $X$. That is, there exist $\delta>0, \varepsilon<0$ and a set $S_{x}^{\prime}$ in $f(\overline{S(x, \delta)},[-\varepsilon, \varepsilon])$ such that for each $y \in f \overline{(S(x, \delta)},[-\varepsilon, \varepsilon])$ there exists a unique $t_{y} \in R$ such that $\left|t_{y}\right| \leqq \varepsilon$ and $f\left(y, t_{y}\right) \in S_{x}^{\prime}$, where $S(x, \delta)$ is a $\delta$-neighborhood of $x$ and $\overline{S(x, \hat{\delta})}$ is the closure of $S(x, \delta)$. Furthermore, $x$ is in $S_{x}^{\prime}$. There is a homeomorphism $h: S_{x}^{\prime} \times[-\varepsilon, \varepsilon] \rightarrow f\left(S_{x}^{\prime},[-\varepsilon, \varepsilon]\right) \subset X$ defined by $h(s, t)=f(s, t), s \in S_{x}^{\prime}, t \in$ $[-\varepsilon, \varepsilon]$.

Let $S_{x}=\left\{f\left(y, t_{y}\right) \in S_{x}^{\prime} \mid y \in S(x, \delta)\right\}$. Then $S_{x} \times(-\varepsilon, \varepsilon)$ is homeomorphic to an open neighborhood of $x$ in $X$. So we may regard $S_{x} \times(-\varepsilon, \varepsilon)$ as a neighborhood of $x$ in $X$. Since the dimension of $S_{x} \times(-\varepsilon, \varepsilon)$ is 1 , the dimension of $S_{x}$ is 0 by [4: p. 222]. Since $S_{x}$ may be taken connected, $S_{x}$ is the point $x$ itself. Hence $(-\varepsilon, \varepsilon)$ is a neighborhood of $x$ in $X$. This proves that each point $x$ in $X$ has an interval neighborhood. Since $X$ is compact, $X$ is a circle.

2. Two dimensional minimal sets. Let $(X, R, f)$ be again a topological transformation group (continuous flow). If a minimal set of $\operatorname{dim}_{L}(X)=2$ is a manifold then it is either a torus or a Klein bottle since its Euler characteristic has to vanish [12: p. 197]. Since a Klein bottle cannot be a minimal set by a result of Kneser [10: p. 153] (we are told this by Arthur J. Schwartz), $X$ must be a torus.

The following seems plausible.

Conjecture. Let $(X, R, f)$ be a topological transformation group with $X$ a locally contractible compact separable metric space. Suppose $X$ is a minimal set and $\operatorname{dim}_{L}(X)=2$. Then $X$ is a manifold, hence a torus. 
If we further assume that $X$ is almost periodic, then $X$ is a homogeneous space [6: p. 343]. By an almost periodic topological transformation group we mean that for given $\varepsilon>0$ there exists a relative dense subset of numbers $\left\{\tau_{n}\right\}$ such that for all $x \in X, d(f(x, t)$, $\left.f\left(x, t+\tau_{n}\right)\right)>\varepsilon$ for all $t \in R$ and each $\tau_{n}$, where $d$ is a complete metric on $X$. A set $Y$ of real numbers is called relative dense if there exists a $T>0$ such that $Y \cap(t-T, t+T) \neq \varnothing$ for all $t \in R$. It is known that such a space $X$ is a torus by a result of [6: p. 39] and Lie group theory. And Bing and Borsuk showed that such a space is a manifold [1: p. 110]. But we give here a proof because the method of Theorem 1 can also be applied to prove this result and we hope that the technique used in the proof is useful to prove that each point $x$ in $X$ has a Euclidean neighborhood without assuming almost periodicity of the action, thus proving the conjecture.

THEorem 2. Let $(X, R, f)$ be a topological transformation group with $X$ a locally contractible compact separable metric space and $R$ the additive real group. Suppose $X$ is an almost periodic minimal set of $\operatorname{dim}_{L}(X)=2$. Then $X$ is a manifold (hence a torus).

Proof. Note again that $X$ is necessarily connected. Since each $x$ in $X$ is not a fixed point, by the section theorem of Bebutov and Hájek there is a section generating arbitrary small neighborhoods of $x$ in $X$. That is, as in Theorem $1, x$ has an open neighborhood of the form $S_{x} \times(-\varepsilon, \varepsilon)$ in $X$. Here a section $S_{x}$ may be taken connected, locally connected and locally compact. Since the dimension of $S_{x} \times(-\varepsilon, \varepsilon)$ is 2, the dimension of $S_{x}$ is at least 1 (in fact, it is 1 [4]). Since $S_{x}$ is locally compact, connected and locally connected, there is a nondegenerate arc $\alpha_{y}$ in $S_{x}$ which contains $y$ for each $y \in S_{x}$. Then $\alpha_{x}[0,1] \times[-\varepsilon, \varepsilon]$ is a closed 2-cell in $X$, and $x \times 0=x \in \alpha_{x}[0,1] \times[-\varepsilon, \varepsilon]$.

Suppose $\alpha_{x}(0,1) \times(-\varepsilon, \varepsilon)$ contains a limit point $x_{0}$ of $X-\left(\alpha_{x}[0,1]\right.$ $[-\varepsilon, \varepsilon])$. Take an open set $V_{0}$ of $x_{0}$ in $\alpha_{x}(0,1) \times(-\varepsilon, \varepsilon)$ such that $\bar{V}_{0}$ is compact and $\bar{V}_{0} \subset \alpha_{x}(0,1) \times(-\varepsilon, \varepsilon)$. Let $V$ be an open neighborhood in $X$ such that $V_{0}=\alpha_{x}(0,1) \times(-\varepsilon, \varepsilon) \cap V$. Since $X$ is locally contractible, there is an open neighborhood $U$ of $x_{0}$ in $X$ such that $U$ is contractible in $V$ to the point $x_{0}$ and $U \cap\left(X-\left(\alpha_{x}[0,1] \times[-\varepsilon, \varepsilon]\right) \neq \varnothing\right.$; i.e., there is a continuous map $H: U \times[0,1] \rightarrow V$ such that $H(y, 0)=$ $y, H(y, 1)=x_{0}$ for each $y \in U$. Then for a point $z \in U \cap\left(X-\left(\alpha_{x}[0,1] \times\right.\right.$ $[-\varepsilon, \varepsilon])), H_{z}:[0,1] \rightarrow V$ is a path from $z$ to $x_{0}$ and $H_{z}[0,1] \cap\left(\alpha_{x}[0,1] \times\right.$ $[-\varepsilon, \varepsilon]) \subset V \cap\left(\alpha_{x}[0,1] \times[-\varepsilon, \varepsilon]\right)=V_{0} \subset \vec{V}_{0} \subset \alpha_{x}(0,1) \times(-\varepsilon, \varepsilon)$. Therefore, there is a path from $x$ to $x_{0}$ which misses $\alpha_{x}[0,1] \times[-\varepsilon, \varepsilon]-$ $\alpha_{x}(0,1) \times(-\varepsilon, \varepsilon)$.

Considering the path is ordered from $z$ to $x_{0}$, there is a point $x_{0}^{\prime} \in \bar{V}_{0}$ such that $x_{0}^{\prime}$ is the first point of the path $H_{z}$ which meets $\bar{V}_{0}$. 
Therefore, there is 2-dimensional umbrella with $x_{0}^{\prime}$ as its center (see [1] for definition). Then each point of $X$ is a center of a 2-dimensional umbrella by the homogeneity of $X$ that follows by the assumptions. This contradicts the umbrella theorem of Bing and Borsuk.

Thus an open 2-disk $\alpha_{x}(0,1) \times(-\varepsilon, \varepsilon)$ is an open set in $X$. If $x \in \alpha_{x}(0,1)$ then $\alpha_{x}(0,1) \times(-\varepsilon, \varepsilon)$ is an open neighborhood of $x$ in $X$. Otherwise to get an open neighborhood of $x$ that is an open 2-disk we appeal to the minimality of $X$ (or homogeneity of $X$ in this case). For if $x$ has no open neighborhood that is an open 2-disk then there is no element of $R$ that sends $x$ into the open set $\alpha_{x}(0,1) \times(-\varepsilon, \varepsilon)$. This contradicts the minimality of $X$.

Therefore, $X$ is a compact 2-manifold. Hence $X$ is a torus by the remark that we made in the beginning of the section.

\section{REFERENCES}

1. R. H. Bing and K. Borsuk, Some remarks concerning topologically homogeneous spaces, Annals of Math., 81 (1965), 100-111.

2. A. Borel, Seminar on transformation groups, Annals of Math. Studies No. 46 (1960), Princeton, N. J.

3. Hsin Chu, Algebraic topology criteria for minimal sets, Proc. Amer. Math. Soc., 13 (1962), 503-508.

4. H. Cohen, A cohomological definition of dimension for locally compact Hausdorff spaces, Duke Math. J., 21 (1954), 209-224.

5. E. E. Floyd, A nonhomogeneous minimal set, Bull. Amer. Math. Soc., 55 (1949), 957-960.

6. W. H. Gottschalk, Minimal sets: An introduction to topological dynamics, Bull. Amer. Math. Soc., 64 (1958), 336-351.

7. W. H. Gottschalk and G. A. Hedlund, Topological Dynamics, Amer. Math. Soc. Colloquium publications, Vol. 36 (1955), Princeton.

8. Otomar Hájek, Sections of dynamical systems in $E^{2}$, Czech. Math. J., 15 (1965), 205-211.

9. J. C. Hocking and G. S. Young, Topology, Addison-Wesley, 1961.

10. H. Kneser, Regulare Kurvenschasen auf den Ringflachen, Mathematische Annalen, 91 (1924), 135-154.

11. V. V. Nemytskii and V. V. Steponov, Qualitative Theory of Differential Equations, Princeton Univ. Press, 1959.

12. E. H. Spanier, Algebraic Topology, McGraw-Hill, 1966.

Received May 27, 1971 and in revised form July 26, 1971.

The University of Connecticut 


\section{PACIFIC JOURNAL OF MATHEMATICS}

\section{EDITORS}

\section{H. SAMElson}

Stanford University

Stanford, California 94305

C. R. Новву

University of Washington

Seattle, Washington 98105
J. DugunduI

Department of Mathematics University of Southern California

Los Angeles, California 90007

RICHARD ARENS

University of California

Los Angeles, California 90024

\section{ASSOCIATE EDITORS}
E. F. BECKENBACH
B. H. NeUmanN
F. WOLF
K. YosHIDA

\section{SUPPORTING INSTITUTIONS}

UNIVERSITY OF BRITISH COLUMBIA

UNIVERSITY OF SOUTHERN CALIFORNIA

CALIFORNIA INSTITUTE OF TECHNOLOGY

STANFORD UNIVERSITY

UNIVERSITY OF CALIFORNIA

UNIVERSITY OF TOKYO

MONTANA STATE UNIVERSITY

UNIVERSITY OF UTAH

UNIVERSITY OF NEVADA

WASHINGTON STATE UNIVERSITY

NEW MEXICO STATE UNIVERSITY

OREGON STATE UNIVERSITY

UNIVERSITY OF OREGON

OSAKA UNIVERSITY

UNIVERSITY OF WASHINGTON

* *

AMERICAN MATHEMATICAL SOCIETY

NAVAL WEAPONS CENTER 


\section{Pacific Journal of Mathematics}

\section{Vol. 43, No. $1 \quad$ March, 1972}

Alexander (Smbat) Abian, The use of mitotic ordinals in cardinal

arithmetic ....................................... 1

Helen Elizabeth. Adams, Filtrations and valuations on rings ......... 7

Benno Artmann, Geometric aspects of primary lattices .............. 15

Marilyn Breen, Determining a polytope by Radon partitions ........... 27

David S. Browder, Derived algebras in $L_{1}$ of a compact group .......... 39

Aiden A. Bruen, Unimbeddable nets of small deficiency .............. 51

Michael Howard Clapp and Raymond Frank Dickman, Unicoherent

compactifications ............................... 55

Heron S. Collins and Robert A. Fontenot, Approximate identities and the strict topology ................................... 63

R. J. Gazik, Convergence in spaces of subsets................. 81

Joan Geramita, Automorphisms on cylindrical semigroups ........... 93

Kenneth R. Goodearl, Distributing tensor product over direct product ..... 107

Julien O. Hennefeld, The non-conjugacy of certain algebras of

operators ................................... 111

C. Ward Henson, The nonstandard hulls of a uniform space ........... 115

M. Jeanette Huebener, Complementation in the lattice of regular

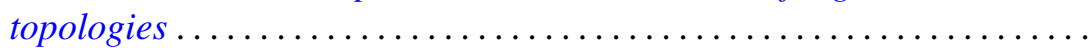

Dennis Lee Johnson, The diophantine problem $Y^{2}-X^{3}=A$ in a

polynomial ring .................................... 151

Albert Joseph Karam, Strong Lie ideals . . . . . . . . . . . . . . . . . . . . 157

Soon-Kyu Kim, On low dimensional minimal sets ............... 171

Thomas Latimer Kriete, III and Marvin Rosenblum, A Phragmén-Lindelöf

theorem with applications to $M(u, v)$ functions ..... . .

William A. Lampe, Notes on related structures of a universal algebra . . . . 189

Theodore Windle Palmer, The reducing ideal is a radical .

207

Kulumani M. Rangaswamy and N. Vanaja, Quasi projectives in abelian and module categories ................................ 221

Ghulam M. Shah, On the univalence of some analytic functions ......... 239

Joseph Earl Valentine and Stanley G. Wayment, Criteria for Banach

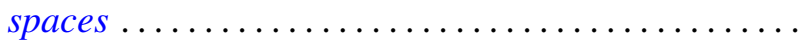

Jerry Eugene Vaughan, Linearly stratifiable spaces ............... 253

Zbigniew Zielezny, On spaces of distributions strongly regular with respect to partial differential operators ..................... 